\title{
Microcirculatory Responses to Adenosine in the Newborn Pig Retina
}

\author{
JEFFREY M. GIDDAY AND T. S. PARK \\ Department of Neurology and Neurological Surgery, and St. Louis Children's Hospital, \\ Washington University School of Medicine, St. Louis, Missouri 63110
}

\begin{abstract}
The reactivity of retinal arterioles and venules to exogenous and endogenous adenosine was investigated in the newborn piglet eye in vivo. The retinal microcirculation of isoflurane-anesthetized newborn pigs was observed at $310 \times$ using videomicroscopy, and changes in the diameter of arterioles $(50-100 \mu \mathrm{m}$ in diameter) and venules (150-250 $\mu \mathrm{m}$ in diameter) occurring in response to intravitreal topical microsuffusions of various adenosinergic compounds were determined. Dose-dependent dilations of the arterioles and venules resulted from intravitreal adenosine $(0.2-200 \mathrm{nmol})$ and three of its agonists: $5^{\prime}-\mathrm{N}$ ethylcarboxyamidoadenosine $(0.2-20 \mathrm{pmol})$, 2-chloroadenosine $(0.2 \mathrm{pmol}-2 \mathrm{nmol})$, and $\mathrm{N}^{6}$-cyclohexyladenosine $(0.2$ and $2.0 \mathrm{nmol}$ ). The resulting order of vasodilatative potency, wherein $5^{\prime}$-N-ethylcarboxyamidoadenosine $\gg>2$ chloroadenosine $>$ adenosine $=\mathrm{N}^{6}$-cyclohexyladenosine, is indicative of $\mathrm{A} 2$ adenosine receptor-mediated vasodilation. Significant arteriolar dilations $[24 \pm 4 \%(p=0.0012)$ and $35 \pm 5 \%(p=0.0008)$, respectively] also resulted from intravitreal application of $0.2 \mathrm{nmol}$ of iodotubercidin, an adenosine kinase inhibitor, and $0.1 \mathrm{nmol}$ of nitrobenzylthioinosine, an inhibitor of adenosine reuptake/transport. The dilation induced by nitrobenzylthioinosine was blocked $(p<0.0004)$ by coadministration of the relatively specific A2 adenosine receptor antagonist 1,3-dipropyl-7methylxanthine ( $1 \mathrm{nmol})$, confirming that nitrobenzylthioinosine induced dilation via potentiation of endogenous adenosine. Administration of 1,3-dipropyl-7-methylxanthine alone did not significantly affect arteriolar or venular diameters, suggesting that endogenous adenosine does not contribute to the maintenance of basal tone. Collectively, these results indicate that vascular $\mathrm{A} 2$ adenosine receptors mediate retinal arteriolar dilation in response to increases in endogenous adenosine concentrations, consistent with our hypothesis that endogenous interstitial adenosine plays an important role in the metabolic regulation of retinal blood flow. (Pediatr Res 33: 620-627, 1993)
\end{abstract}

Abbreviations
RBF, retinal blood flow
$\mathrm{MABP}$, mean arterial blood pressure
$\mathrm{PaO}_{2}$, arterial $\mathrm{O}_{2}$ tension
$\mathrm{PaCO}_{2}$, arterial $\mathrm{CO}_{2}$ tension
$\mathrm{NECA}, 5^{\prime}$-N-ethylcarboxamidoadenosine
2CADO, 2-chloroadenosine
CHA, N6 -cyclohexyladenosine
ITC, iodotubercidin

Received November 4, 1992; accepted January 26. 1993

Correspondence and reprint requests: Jeffrey M. Gidday, Ph.D., Department of Neurosurgery. Room 1S-46. St. Louis Children's Hospital, One Children's Place. St. Louis. MO 63110

Supported by a grant from the Lucille P. Markey Foundation.
NBTI, 4-nitrobenzyl-6-thioinosine DPMX, 1,3-dipropyl-7-methylxanthine

Despite decades of empirical studies of retinal vascular reactivity, little is known regarding the underlying physiologic mechanisms controlling RBF. Because the microcirculation of the retina is not innervated by the autonomic nervous system and because it exhibits a high oxygen extraction under basal conditions, it is likely that metabolic regulation of blood flow predominates in this tissue (1). However, studies demonstrating changes in $\mathrm{RBF}$ in response to changes in putative vasoactive metabolites are surprisingly rare.

We have developed an in vivo retinal microcirculation model in the newborn pig, wherein we can examine changes in the caliber of retinal arterioles and venules in response to local intravitreal injections of drugs and/or systemic interventions. Our broad objective is to test the hypothesis that the purine vasodilator metabolite adenosine participates in the regulation of RBF. Formed as a result of ATP hydrolysis concomitant with neuronal activity, interstitial adenosine is an ideal metabolite to mediate the local coupling of retinal metabolism and retinal flow. In fact, there is now extensive literature from our laboratory and others supporting the role of adenosine in the metabolic regulation of cerebral blood flow in both newborn and adult animals [see Phillis (2) for review]. However, investigations of the effects of adenosine on RBF indices are few $(3,4)$. Adenosine's vascular actions in the eye of the newborn animal are not known.

In the present report, we describe results of experiments designed to test the general hypothesis that the newborn retinal circulation is responsive to adenosine, a prerequisite for further investigations of adenosine's participation in the metabolic regulation of RBF. In particular, the hypothesis predicts that the resistance vessels of the retina should possess adenosine receptors that transduce dose-dependent vasodilatative responses when activated by exogenously administered adenosine and its vasoactive agonists. Secondly, local administration of drugs known to increase interstitial adenosine concentrations should result in arteriolar vasodilation if endogenous interstitial adenosine is vasoactive in the retina. In addition, drugs that inhibit adenosine action should block these responses if administered concomitantly, and, when administered alone, should decrease arteriolar diameter if adenosine contributes to the maintenance of baseline tone.

\section{MATERIALS AND METHODS}

Surgical Preparation. The studies herein were approved by the Animal Studies Committee at Washington University and were in accordance with the Guide for the Care and Use of Laboratory Animals (US Department of Health and Human Services, NIH Publication No. 86-23). 
Newborn piglets (1-2 kg; less than $3 \mathrm{~d}$ of age) were premedicated, tracheotomized, and mechanically ventilated. They were anesthetized with $1.0-1.5 \%$ isoflurane (1 MAC), a level typically requiring no further adjustment during the experiment. A femoral catheter was placed in the artery for regular determination of blood gas variables (Corning model 158 blood gas analyzer, Ciba Corning Diagnostics Corp., Medfield, MA), hematocrit, glucose concentration (YSI blood glucose analyzer, Yellow Springs Instrument Co., Yellow Springs, $\mathrm{OH}$ ), and arterial pressure recording (Gould RS 3400 recorder, Gould, Inc., Cleveland, $\mathrm{OH})$; a femoral venous catheter allowed for maintenance of body fluid balance ( $5 \%$ dextrose in saline; $5 \mathrm{~mL} / \mathrm{kg} / \mathrm{h}$, i.v.). End-tidal $\mathrm{CO}_{2}$ was continuously monitored (Hewlett-Packard $78356 \mathrm{~A}$ Capnometer, Hewlett-Packard Co., Palo Alto, CA). Rectal temperature was maintained at $39^{\circ} \mathrm{C}$ with a thermoregulated heating pad.

The animal's head was stabilized within a customized aluminum cradle held within a stereotaxic frame so that one eye was positioned horizontally for overhead videomicroscopy. The eyelids were gently retracted by surgical staples, and, after dilation of the pupil with $1 \%$ tropicamide, pancuronium bromide was administered $(0.5-\mathrm{mg} / \mathrm{kg}$ intramuscular loading dose; $0.25-\mathrm{mg} /$ $\mathrm{kg} / \mathrm{h}$ i.v. maintenance dose) to keep the extraocular muscles immobilized during the experimental period. For optically undisturbed viewing of the retinal microcirculation, an artificial anterior chamber, similar to that used by Flower and Patz (5), was used. After excision of the cornea with a 7-mm trephine and removal of the lens, a $12-\mathrm{mm}$ round glass coverslip (no. 0; VWR Scientific, Westchester, PA) was placed on the surface of the undisturbed posterior tunica vasculosa lentis to allow for stereomicroscopic observation of the fundus. With this procedure, no vitreous was lost, and retinal detachment was never observed. Unlike Flower and Patz (5), however, we did not glue the cover glass to the eye, so intraocular pressure lost as a result of the lensectomy was not reestablished. After this surgical procedure, $30 \mathrm{~min}$ were allowed before initiation of all experimental protocols.

Videomicroscopy. The retinal arterioles and venules lying superficially on the piglet fundus were observed by using a stereomicroscope (Olympus SZ-6045, Olympus, New Hyde Park, New York) mounted on a flexible boom stand, with true axial illumination provided by a xenon fiberoptic light source (model 611 , K. Storz, Culver City, CA) and a custom-designed intramicroscopic reflecting mirror. Images were processed on-line for optimal contrast (Hamamatsu Argus- 10 image processor, Hamamatsu Corp., Middlesex, NJ), monitored on a high-resolution video monitor (Sony PVM1342Q), and captured on videotape (JVC Super VHS model HR-S8000U) using a newvicon tube camera (Panasonic WV-1550). A digital timer with 10-ms resolution, provided by the image processor, was input into the videocassette recorder to document the durations of various microcirculatory responses. With the optomechanical coupling of the stereomicroscope photo-eyepiece, the extender tube, and the electronic magnification inherent in the camera, $310 \times$ magnification was achieved for videorecording. This allowed for determinations of the diameters of an adjacent arteriolar (50- to $100-\mu$ m diameter) and venular (100- to $250-\mu \mathrm{m}$ diameter) pair, typically in the superior quadrant (6), lying within one disc diameter of the optic disc. Measurements were undertaken manually with a mouse-driven cursor, either on-line (Hamamatsu Argus-10 image processor) or off-line (Bioscan "Optimas" Image Analyses Systems, Bioscan, Inc., Edmonds, WA); repeated measurements indicated $\pm 2-\mu \mathrm{m}$ accuracy for arterioles of the size typically encountered in our studies. Changes in arteriolar diameter occurring under conditions of constant retinal perfusion pressure (MABP) were used in this study as indices of changes in RBF.

Drug Administrations. Adenosine agonists and other compounds were dissolved in the necessary solvent (see below) and diluted at least 100-fold with sterile balanced salt solution (BSS+;
Alcon Surgical, Inc., Fort Worth, TX) to achieve the final desired concentrations. The $0.22-\mu \mathrm{m}$-filtered drug solutions were gassed with $95 \% \mathrm{~N}_{2} / 5 \% \mathrm{CO}_{2}$ to achieve $\mathrm{pH}$ and gas tensions similar to those found in the preretinal vitreous $\left[\mathrm{pH}=7.37-7.43, \mathrm{PaO}_{2}=\right.$ $20-40 \mathrm{~mm} \mathrm{Hg}(2.7-5.3 \mathrm{kPa})$, and $\mathrm{PaCO}_{2}=30-40 \mathrm{~mm} \mathrm{Hg}(4.0$ $5.3 \mathrm{kPa})$ ]. The solution was then "microsuffused" onto the abluminal surface of the vessel pair by a localized intravitreal injection, as follows: The blunted tip of a 30-gauge needle, attached to a micromanipulator-mounted, gas-tight, $100-\mu \mathrm{L}$ syringe (Hamilton Company, Reno, NV), was inserted through the lensectomy opening and then advanced to within $50-100 \mu \mathrm{m}$ of the surface of the juxtaposed vessels. A $20-\mu \mathrm{L}$ aliquot of drug solution was then injected over a period of $30 \mathrm{~s}$.

We quantified the vasoactive effect of all drugs in two ways: l) the maximum change in arteriolar or venular diameter, as determined by the mean of the four largest changes in diameter measured over the entire period of vessel observation, and 2) the integrated area under the resultant diameter versus time response curve; this latter measurement therefore collectively included the duration of the vasoactive response as well as its intensity (maximum diameter change).

Protocols. Validation of microcirculatory reactivity. MABP (normal range $60-75 \mathrm{~mm} \mathrm{Hg}$ ) and blood gas parameters are very stable in this preparation, allowing for at least $3 \mathrm{~h}$ of steady state observation of the retinal microcirculation. We verified in four animals that significant changes in arteriolar $(65 \pm 14 \mu \mathrm{m})$ and venular $(136 \pm 34 \mu \mathrm{m})$ diameters do not occur during a 180-min period of observation (by analysis of variance). The temperature of the preretinal vitreous, measured simultaneously with a 25 gauge temperature probe advanced to the vitreous-retina boundary, also did not vary significantly during this time, remaining at $38.1 \pm 0.1^{\circ} \mathrm{C}$ (with a corresponding rectal temperature of $37.8 \pm 0.1^{\circ} \mathrm{C}$ ). Thus, no potential temporal changes in our index of $\mathrm{RBF}$ occur in this preparation due to drift, xenon light exposure, or cumulative changes in retinal temperature. All experimental protocols described herein were completed within $180 \mathrm{~min}$

Given that endogenous intraocular pressure was lost with the lensectomy procedure used herein, we deemed it necessary to demonstrate that the caliber of the resistance vessels in this preparation respond appropriately to various systemic interventions. Changes in systemic oxygenation (hypoxia and hyperoxia), changes in $\mathrm{PaCO}_{2}$ (hypercapnia and hypocapnia), and reductions in retinal perfusion pressure induced by systemic hemorrhagic hypotension (a reduction in normotensive MABP from $69 \pm 1$ $\mathrm{mm} \mathrm{Hg}$ to $38 \pm 1 \mathrm{~mm} \mathrm{Hg}$ ), were used as stimuli to assess expected microcirculatory reactivity, each in separate animal groups (Table 1).

In all studies described herein, stable levels of baseline arteriolar and venular tone were always documented before initiation of a particular stimulus or intravitreal administration of a given drug. We operationally defined stable tone as a $< \pm 5 \%$ change in arteriolar diameter measured intermittently (typically at 10-min intervals) over a minimum duration of $20 \mathrm{~min}$. Aside from the above-mentioned studies in which $\mathrm{PaO}_{2}, \mathrm{PaCO}_{2}$, and systemic blood pressure were intentionally changed, experiments in which significant changes in these variables were observed were not included in the analyses, given the dramatic influence such changes can have on RBF.

Dose-response studies of adenosine and adenosine agonists. Adenosine, or one of three adenosine agonists, were microsuffused onto the retinal microcirculation to determine its responsiveness to these compounds. Because in many cases the arteriolar dilations ensuing from these adenosinergic compounds were long-lasting, only one drug, at one concentration, was tested in each animal. The diameters of the retinal arteriole and venule were measured $5,10,15,20,30,40,60,80,100,120,140,160$, and $180 \mathrm{~min}$ after local intravitreal microsuffusion $(20 \mu \mathrm{L})$ of each drug.

The injected doses of adenosine were $0.2,2,20$, and $200 \mathrm{nmol}$. 
Table 1. Validation of retinal microcirculatory reactivity*

\begin{tabular}{|c|c|c|c|c|c|c|}
\hline \multirow[b]{3}{*}{ Stimulus } & & & \multicolumn{4}{|c|}{ Percent change in vessel diameter } \\
\hline & \multicolumn{2}{|c|}{ Initial diameter $(\mu \mathrm{m})$} & \multicolumn{2}{|c|}{ Stimulus } & \multicolumn{2}{|c|}{ Poststimulus } \\
\hline & Arteriole & Venule & Arteriole & Venule & Arteriole & Venule \\
\hline $\begin{array}{l}\text { Hypoxia } \\
\quad 30 \mathrm{~min} ; n=4 \\
\mathrm{PaO}_{2}=26 \pm 2 \mathrm{~mm} \mathrm{Hg}(3.5 \pm 0.3 \mathrm{kPa})\end{array}$ & $70 \pm 5$ & $173 \pm 48$ & $31 \pm 6 \dagger$ & $31 \pm 13$ & $5 \pm 4$ & $-1 \pm 4$ \\
\hline $\begin{array}{l}\text { Hyperoxia } \\
\quad 60 \mathrm{~min} ; n=6 \\
\mathrm{PaO}_{2}=398 \pm 34 \mathrm{~mm} \mathrm{Hg}(53.1 \pm 4.5 \mathrm{kPa})\end{array}$ & $71 \pm 5$ & $171 \pm 15$ & $-7 \pm 3 \dagger$ & $-19 \pm 3 \dagger$ & $3 \pm 2$ & $-5 \pm 4$ \\
\hline $\begin{array}{l}\text { Hypercapnia } \\
\qquad 15 \mathrm{~min} ; n=6 \\
\mathrm{PaCO}_{2}=80 \pm 5 \mathrm{~mm} \mathrm{Hg}(10.7 \pm 0.7 \mathrm{kPa}) \\
\mathrm{pH}=7.08 \pm 0.05\end{array}$ & $81 \pm 4$ & $150 \pm 8$ & $27 \pm 3 \dagger$ & $43 \pm 7 \dagger$ & $3 \pm 2$ & $16 \pm 6$ \\
\hline $\begin{array}{l}\text { Hypocapnia } \\
20 \mathrm{~min} ; n=3(2.8 \pm 0.4 \mathrm{kPa}) \\
\mathrm{PaCO}_{2}=21 \pm 3 \mathrm{~mm} \mathrm{Hg} \\
\mathrm{pH}=7.68 \pm 0.08\end{array}$ & $81 \pm 4$ & $208 \pm 11$ & $-10 \pm 1 \dagger$ & $-12 \pm 3 \dagger$ & $-4 \pm 1 \dagger$ & $-23 \pm 5 \dagger$ \\
\hline $\begin{array}{l}\text { Hypotension } \\
\qquad 60 \mathrm{~min} ; n=5 \\
\mathrm{MABP}=38 \pm 1 \mathrm{~mm} \mathrm{Hg}\end{array}$ & $74 \pm 2$ & $201 \pm 30$ & $27 \pm 5 \dagger$ & $3 \pm 7$ & $7 \pm 5$ & $-11 \pm 19$ \\
\hline
\end{tabular}

* Data are mean \pm SEM. Vessel diameter measurements were obtained at 5- or 10-min intervals throughout the intervention period and at similar intervals after cessation of the stimulus. Changes in vessel diameter were quantified by normalizing the diameter change at each time point to the mean of the initial baseline diameter (shown in $\mu \mathrm{m}$ ); the mean change in diameter during the intervention and poststimulus periods is indicated.

$\dagger$ Significantly different from initial baseline diameter, $p<0.05$ (paired $t$ test).

For the agonists, the injected doses were $0.2,2$, and 20 pmol of NECA; 2 pmol, 20 pmol, 200 pmol, and 2 nmol of 2CADO; and 0.2 and $2 \mathrm{nmol}$ of CHA. Stock solutions of adenosine, NECA, and 2CADO were prepared in saline and diluted at least 100 -fold with BSS+. BSS+ alone had no significant effect on retinal vessel tone; in seven animals (resting retinal arteriolar diameter $73 \pm 6 \mu \mathrm{m}$; retinal venular diameter $142 \pm 22 \mu \mathrm{m}$ ), a $20-\mu \mathrm{L}$ microsuffusion of BSS + had no significant vasoactive effect on these vessels $(1.5 \pm 1.7 \%$ and $1.2 \pm 1.5 \%$ increase in diameter, respectively). CHA was prepared at $100 \mathrm{mM}$ in methanol and then diluted to 10 and $100 \mu \mathrm{M}$ with BSS+; we documented in separate studies ( $n=4$ animals) that the resultant vehicles $(0.1$ and $0.01 \%$ methanol) were without significant vasoactive effect; administration of the $0.1 \%$ methanol vehicle caused a $2.6 \pm 1.2 \%$ dilation of arterioles $(71 \pm 11 \mu \mathrm{m})$, and a $1.4 \pm 0.6 \%$ dilation of venules $(176 \pm 24 \mu \mathrm{m})$.

Pharmacologic potentiation of endogenous interstitial adenosine. We assessed the effects of intravitreal microsuffusion of 0.2 nmol of ITC or $0.1 \mathrm{nmol}$ of NBTI. An increase in interstitial adenosine concentrations is expected to result from administration of each of these drugs, but by different mechanisms of action. ITC inhibits adenosine kinase, the enzyme responsible for the conversion of adenosine to AMP, and NBTI inhibits adenosine reuptake into retinal neurons, endothelial cells, and glia. As with the adenosine analogs, drug-induced changes in the diameters of the retinal arterioles and venules were monitored at regular intervals for $180 \mathrm{~min}$ after local administration, and quantification of the dilatative response was carried out in an identical fashion. ITC and NBTI were dissolved at $10 \mathrm{mM}$ in DMSO and diluted with BSS+ to the desired final concentrations. Assessments of the potential dilatative effect of the resultant vehicles (0.1\% DMSO for ITC and $0.05 \%$ DMSO for NBTI) were undertaken in four separate animals, but no vasoactivity was evidenced; with $0.1 \%$ DMSO, a $-0.6 \pm 1.8 \%$ change in diameter occurred in observed arterioles $(6 \overline{5} \pm 4 \mu \mathrm{m})$ and a $0.4 \pm 3.1 \%$ change in diameter occurred in venules (182 \pm 24 $\mu \mathrm{m})$.

Effects of adenosine receptor blockade. The effect of local administration of the A2 selective adenosine antagonist DPMX was also determined. DPMX ( $1 \mathrm{nmol}$ in $0.05 \%$ DMSO solvent) was administered alone in four animals and in conjunction with
$0.1 \mathrm{nmol}$ of NBTI in four additional animals, and diameter changes were measured at 10 -min intervals for $120 \mathrm{~min}$ after drug administration.

Statistics. All results are shown as mean \pm SEM. Paired or unpaired $t$ tests were used, with Bonferroni corrections when appropriate, to identify significant changes in vessel diameters in response to systemic interventions or intravitreal microsuffusions of drugs. Vasoactive responses to the appropriate vehicle served as the statistical control for each drug. Significance was tested at the $95 \%$ confidence interval.

\section{RESULTS}

Validation of microcirculatory reactivity. Induction of systemic hypoxia, hyperoxia, hypercapnia, hypocapnia, or hypotension led to significant changes in retinal arteriolar tone. The results of these studies are shown in Table 1. For all interventions, significant changes in arteriolar diameter were measured within 5 min of inducing the vasoactive stimulus, and the new level of tone achieved was then sustained at relatively constant levels until the cessation of the stimulus; 10 min thereafter, arteriolar tone had returned to levels (designated "Poststimulus" in Table 1) that were generally not significantly different from those measured during baseline conditions. No significant changes in MABP occurred with the hypoxic, hypercapnic, and hypocapnic stimuli; a small but significant decrease in MABP was noted with hyperoxia, which may have offset slightly the extent of vasoconstriction evidenced in response to this stimulus.

Adenosine and adenosine agonists: dose response. Complete dose-response curves were not obtained for adenosine and its agonists, in terms of identifying maximal responses; thus, a true $\mathrm{ED}_{50}$ could not be calculated. Nevertheless, based on the data obtained, differences in relative potencies of adenosine and its agonists were obvious, as is evident in Figure 1. The mean values resulting from integrating the area under the percent arteriolar dilation versus time response curve for each drug concentration examined are plotted in this figure, thereby incorporating both the intensity and the duration of each drug-induced vasoactive response.

In general, intravitreal microsuffusions of adenosine and each of its agonists resulted in significant dose-dependent dilations of 


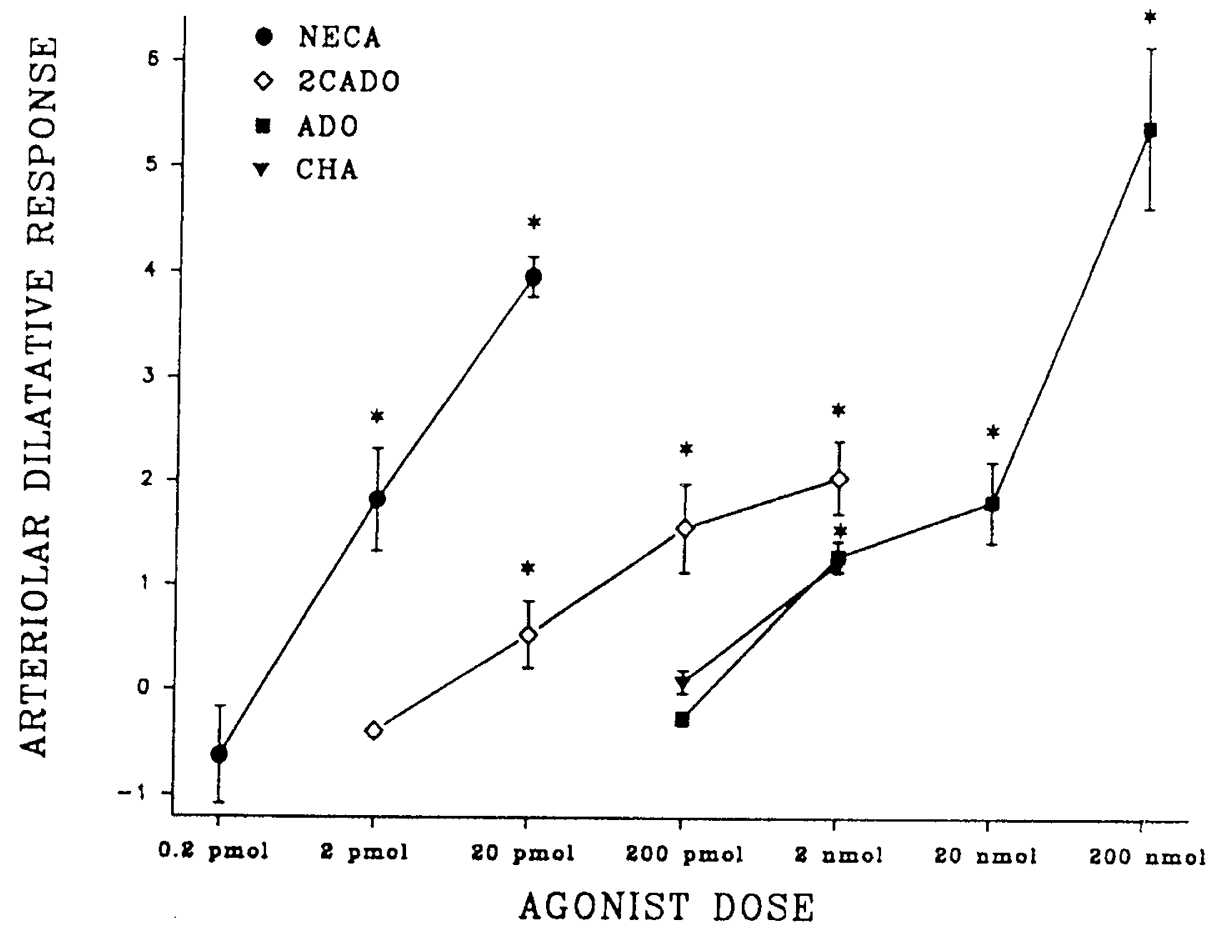

Fig. 1. Retinal arteriolar dose response to adenosine and its agonists. Dose-response relations indicating the extent of retinal arteriolar dilation induced by intravitreal microsuffusion of different doses of NECA, 2CADO, adenosine $(A D O)$, and CHA. The responses were quantified by integrating the area under the dilation $v s$ time response curve for each drug concentration (see text); thus, the ordinate (in arbitrary units) reflects both the duration of the response as well as its intensity (shown separately in Fig. 2). *, Significantly greater than dilations induced by the respective vehicle; $p<0.05$; unpaired $t$ test.

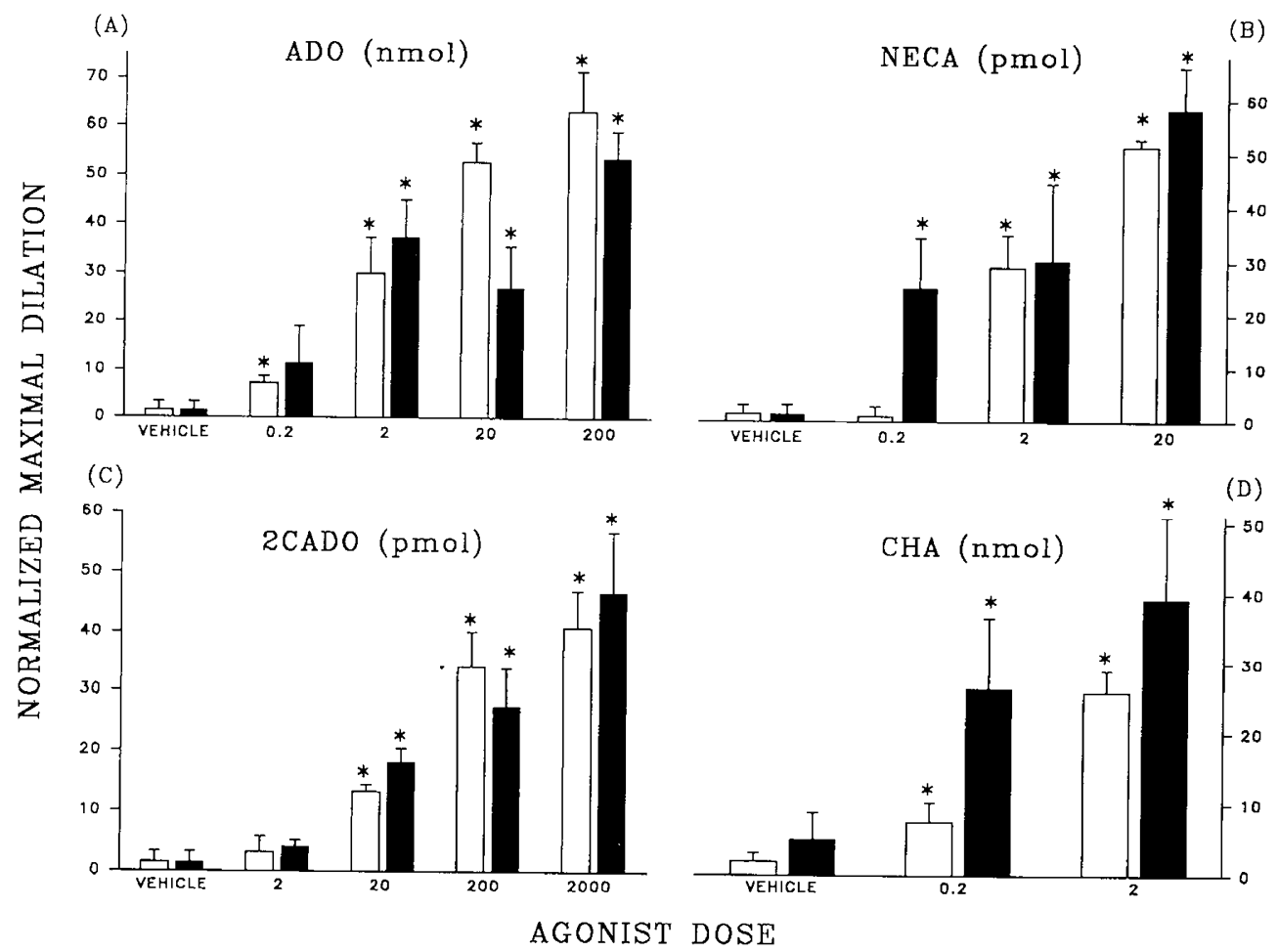

Fig. 2. Retinal microvessel dilations induced by adenosine and its agonists. Effect of intravitreally microsuffused adenosine $(A D O)(A), \mathrm{NECA}$ $(B), 2 \mathrm{CADO}(C)$, and CHA $(D)$ on diameters of retinal arterioles (open bars) and venules (filled bars). The effect of each drug concentration was tested in separate animals (see text). Percent changes in diameter were obtained by normalizing the drug-induced changes in vessel diameter to the steady state diameters measured during basal conditions before intravitreal microsuffusion of the particular drug or vehicle; the maximal change in diameter induced by each drug is shown. *, Significantly different from baseline diameter; $p<0.05$; paired $t$ test. 
both arterioles and venules (Figs. 1 and 2). In the animals in which adenosine reactivity was assessed, baseline arteriolar and venular diameters were $82 \pm 3 \mu \mathrm{m}(n=12)$ and $167 \pm 12 \mu \mathrm{m}$ $(n=12)$, respectively. Resting arteriolar diameters for those animals in which NECA, 2CADO, and CHA reactivity were determined were $77 \pm 4 \mu \mathrm{m}(n=11), 78 \pm 4 \mu \mathrm{m}(n=14)$, and $96 \pm 3 \mu \mathrm{m}(n=6)$, respectively; baseline venular diameters for the same groups were $157 \pm 13 \mu \mathrm{m}(n=11), 164 \pm 13 \mu \mathrm{m}(n=$ $14)$, and $170 \pm 14 \mu \mathrm{m}(n=6)$, respectively.

A 0.2 -nmol dose of adenosine $(n=3)$ dilated arterioles significantly $(7 \pm 2 \%)$; the observed venular dilation $(12 \pm 7 \%)$ at this dose, however, did not reach significance (Fig. $2 A$ ). Microsuffusions of $2 \mathrm{nmol}(n=3), 20 \mathrm{nmol}(n=3)$, and $200 \mathrm{nmol}(n=3)$ of adenosine led to significant dilations of $30 \pm 7 \%, 53 \pm 4 \%$, and $63 \pm 8 \%$, respectively, in the arterioles and $37 \pm 8 \%, 27 \pm$ $8 \%$, and $54 \pm 6 \%$, respectively, in the venules. The maximal arteriolar dilatative effect occurred $20 \pm 4 \min (n=12$; all doses tested) after local administration. The duration of the adenosineinduced arteriolar dilation was also related proportionally to dose; for the four doses examined, the dilatative response lasted roughly $60,80,100$, and $>180 \mathrm{~min}$ (in the case of the 200-nmol dose).

NECA was the most potent adenosine agonist we studied (Fig. $2 B$ ), roughly 5000-10000 times more potent than adenosine or $\mathrm{CHA}$, and 100 times more potent than 2CADO. Microsuffusion of 0.2 pmol $(n=3), 2$ pmol $(n=4)$, and 20 pmol $(n=4)$ of NECA caused arteriolar dilations of $1 \pm 2 \%, 29 \pm 6 \%$, and 51 $\pm 1 \%$, respectively; the latter two responses were significant. Significant venular dilations of $25 \pm 9 \%, 30 \pm 14 \%$, and $58 \pm$ $8 \%$ were concomitantly observed with these three doses. Fiftytwo $\pm 10 \min (n=11)$ were required for this agonist to exhibit its maximal dilatative effect in the arterioles. The dilation ensuing from the 2-pmol dose lasted approximately $120 \mathrm{~min}$; that resulting from the 20 -pmol dose lasted approximately $180 \mathrm{~min}$. The integrated dilatative response measured in the arterioles in response to NECA is shown in Figure 1.

2CADO also led to dose-dependent arteriolar dilations (Fig. 2C). 2CADO was 10-100 times more potent than adenosine or $\mathrm{CHA}$ at inducing arteriolar dilations. Microsuffusion of $2 \mathrm{pmol}$ of $2 \mathrm{CADO}(n=3)$ was not sufficient to elicit a significant dilation of arterioles $(3 \pm 3 \%$ increase in diameter) or venules $(4 \pm 1 \%)$ relative to vehicle-induced responses. Twenty pmol $(n=3), 200$ pmol $(n=5)$, and 2000 pmol $(n=3)$ of 2CADO caused significant arteriolar dilations of $13 \pm 1 \%, 34 \pm 6 \%$, and $41 \pm$ $6 \%$, respectively; venules significantly increased in diameter by $18 \pm 2 \%, 28 \pm 5 \%$, and $47 \pm 10 \%$, respectively, in response to these doses of 2 CADO. Thirty-two $\pm 7 \mathrm{~min}(n=14)$ elapsed postmicrosuffusion before the maximum dilatative effect was observed; dilations for all doses tested tended to last for only 120 $\min$. The integrated dilatative response in the arterioles resulting from the 2CADO microsuffusions is shown in Figure 1.

$\mathrm{CHA}$ was the least potent of the adenosine agonists, approximately equipotent with adenosine at the two doses tested (Fig. $2 D$ ); solubility problems precluded the assessment of higher concentrations of $\mathrm{CHA}$. An $8 \pm 3 \%$ and $26 \pm 3 \%$ increase in arteriolar diameter occurred in response to $0.2 \mathrm{nmol}(n=3)$ and $2 \mathrm{nmol}(n=3)$ of $\mathrm{CHA}$, respectively. The venular dilations were more pronounced; a $26 \pm 10 \%$ and $39 \pm 12 \%$ increase in diameter resulted from these respective doses. The maximum dilatative effect occurred $25 \pm 5 \min (n=6)$ after drug administration; the duration of the dilation was relatively short, however, with baseline diameters reestablished within $80 \mathrm{~min}$. Figure 1 illustrates the CHA-induced integrated arteriolar response relative to adenosine and the other agonists.

Potentiation of endogenous interstitial adenosine. Both ITC and NBTI, drugs that were expected to cause increases in extracellular adenosine concentrations, caused significant dilations of the retinal microcirculation after intravitreal microsuffusion (Fig. 3). Specifically, $0.2 \mathrm{nmol}$ of ITC $(n=5)$ caused a $24 \pm 4 \%$ ( $p=$ $0.0012)$ and $20 \pm 6 \%(p=0.03)$ increase in arteriolar $(87 \pm 7$ $\mu \mathrm{m})$ and venular diameter $(205 \pm 22 \mu \mathrm{m})$, respectively. The arteriolar dilations induced by ITC reached a maximum $36 \pm$ $10 \mathrm{~min}$ after drug delivery and were fairly long-lasting ( $144 \pm 19$ $\mathrm{min}$ ) relative to the durations of the dilations induced by adenosine and its agonists; in three of these animals, the observed dilatative response was still significant ( $18 \pm 4 \%$ above baseline diameter) $180 \mathrm{~min}$ after ITC administration. Microsuffusion of $0.1 \mathrm{nmol}$ of NBTI $(n=4)$ caused a $35 \pm 5 \%(p=0.0008)$ and $24 \pm 6 \%(p=0.015)$ increase in arteriolar $(70 \pm 7 \mu \mathrm{m})$ and venular $(176 \pm 25 \mu \mathrm{m})$ diameters. The maximal response was noted at $30 \pm 7$ min postadministration, and the dilation lasted $130 \pm 21 \mathrm{~min}$. The integrated dilatative responses to both drugs are shown in Figure 3.

Adenosine receptor blockade. Coadministration of $1 \mathrm{nmol}$ $(n=4)$ of the preferential A2 adenosine receptor antagonist DPMX significantly blocked the arteriolar $(84 \pm 2 \mu \mathrm{m})$ dilatative response to 0.1 nmol of NBTI (Fig. 3); the maximal increase in arteriolar diameter typically resulting from NBTI administration $(35 \pm 5 \%)$ was blocked $86 \%(p=0.002)$ by DPMX (the maximal dilation observed with concomitant administration was only $5 \pm 2 \%$ above baseline). Based on the integrated arteriolar dilatative responses (to thereby include the reduction in the duration of the NBTI-induced dilation), DPMX blocked the dilatative response to NBTI by $92 \%(p=0.0004)$. With respect to the venular $(178 \pm 8 \mu \mathrm{m})$ response, the maximum NBTIinduced venular dilation $(24 \pm 6 \%)$ was also attenuated $(63 \%$ blockade), but the change was not statistically significant ( $p=$ 0.097); similarly, the attenuative effect of DPMX on the integrated venular response did not reach significance $(p=0.074)$.

When $1 \mathrm{nmol}$ of DPMX was microsuffused alone $(n=4)$, no significant change in resting arteriolar $(76 \pm 7 \mu \mathrm{m})$ or venular $(197 \pm 30 \mu \mathrm{m})$ tone $(3 \pm 3 \%$ and $6 \pm 2 \%$ increase in diameters, respectively) was observed (Fig. 3). We confirmed that the retinal vascular bed was still reactive in these animals, as a subsequent 20-min hyperoxic challenge $\left[\mathrm{PaO}_{2}=382 \pm 31 \mathrm{~mm} \mathrm{Hg}(51.0 \pm\right.$ $4.1 \mathrm{kPa}$ )] caused retinal arterioles and venules to constrict $8 \pm$ $3 \%$ and $14 \pm 4 \%$, respectively, similar to the constriction observed in other animals (Table 1).

\section{DISCUSSION}

The present study has demonstrated, in the newborn piglet retina, that: $l$ ) the microcirculation responds with dose-dependent dilations to intravitreal microsuffusions of adenosine and three of its agonists; 2) the resultant order of agonist potency indicates that the observed arteriolar vasodilation is mediated through A2 adenosine receptor activation; 3) dilations of the microcirculatory vessels occur in response to intravitreal ITC and NBTI, drugs known to increase endogenous interstitial adenosine concentrations; and 4) blockade of A2 adenosine receptors with the preferential antagonist DPMX does not affect resting arteriolar or venular tone. These adenosine reactivity studies suggest that, although endogenous adenosine may not exert a tonic vasodilatative effect on the retinal microcirculation, increases in its interstitial concentration can induce arteriolar vasodilation. These findings are consistent with a role for this purine in the metabolic regulation of $\mathrm{RBF}$ in the eye of the newborn.

These data are the first to document adenosine's vasoactive effects in the retina of a newborn animal. In two studies of adult animals $(3,4)$, intravitreal adenosine also elicited an increase in RBF (4) and an increase in retinal vein diameters (3) in the rabbit $(3,4)$, cat $(3)$, and marmoset (3). In neither study was the temporal nature of the vasoactive response documented as in the present report, wherein the arteriolar and venular dilations induced by microsuffusion of adenosine close to the abluminal surfaces of the retinal vessels reached a maximum within $20 \pm 4$ min and lasted less than $2 \mathrm{~h}$, with the exception of the dilatative response to the 200-nmol dose, which was still significantly above baseline $(18 \pm 6 \%)$ at $3 \mathrm{~h}$ postinjection. These findings are in 
3.0

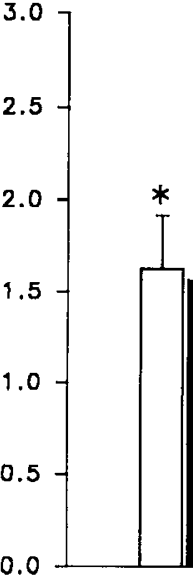

*

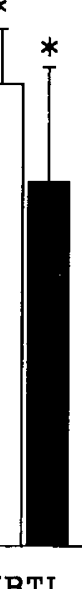

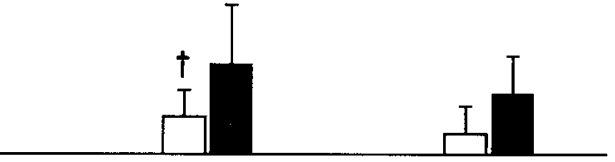

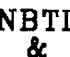

DPMX

Fig. 3. Pharmacologic manipulation of endogenous retinal adenosine. The effect of intravitreal microsuffusions $0.2 \mathrm{nmol}$ of ITC, $0.1 \mathrm{nmol}$ of NBTI, $0.1 \mathrm{nmol}$ of NBTI coadministered with $1 \mathrm{nmol}$ of DPMX, and $1 \mathrm{nmol}$ of DPMX alone on diameters of retinal arterioles (open bars) and venules ( $f$ illed bars). Dilatative responses were quantified by integration as described in Figure 1. *, Significantly greater than the dilations induced by the respective vehicle; $p<0.05$; unpaired $t$ test. $\dagger$, Significantly different from the dilation induced by NBTI alone; $p<0.05$; unpaired $t$ test.

concert with the general observation in brain $(7,8)$ and retina $(9-11)$ that extracellular adenosine is rapidly taken up by neuronal, glial, and endothelial cells, in addition to being metabolized to vasoinactive inosine by adenosine deaminase (12). This lability not only explains, in part, the more potent vasodilatative responses to the slowly metabolizable synthetic adenosine agonists, but also provides the necessary metabolic and compartmental basis for a localized and rapidly responding RBF regulation system responsive to changes in endogenous interstitial fluid adenosine concentrations.

Adenosine receptor pharmacology for a given vascular bed is typically investigated by a ranking of the vasodilatative potencies of synthetic adenosine analogs with differential selectivity for the two primary adenosine receptor subtypes, termed A1 and A2. NECA has been used as a prototypical A2 adenosine agonist in studies designed to elucidate whether the A2 receptor mediates a particular adenosine-evoked response (13). The vasodilatative response profile depicted in Figure 1, wherein NECA $\gg$ $2 \mathrm{CADO}>\mathrm{ADO}=\mathrm{CHA}$, indicates that retinal microcirculatory dilation is mediated by $\mathrm{A} 2$ adenosine receptors. This finding is consistent with the preferential localization of A2 adenosine receptors on vascular smooth muscle, as similar potency profiles were found to characterize the cerebral vasculature of the adult (14) and newborn (15) pig and other animals $(16,17)$. In the adult rabbit retina, similar pharmacologic evidence has been provided indicating A2 adenosine receptor-mediated vasodilation (3).

Our observation of a 5000-10 000-fold greater vasodilatative potency of NECA compared with adenosine is unique in that the potency difference noted in rabbit retina in vivo was only slightly greater than 10-fold (3). Other than differences in species and age, we cannot offer a reason for such a difference, as the only other in vivo study documenting such a comparison in the vasculature of CNS tissue also found, as we did, that NECA was much more potent (500-fold) than adenosine in dilating the rat pial microcirculation (16). Our finding that 2CADO was 10-100 times less potent than NECA in eliciting a vasodilatative response reflects the lower affinity that $2 \mathrm{CADO}$ typically exhibits for the A2 receptor subtype. The greater potency of both these agonists relative to adenosine results not only from their subtype specificity but also because they are relatively resistant to enzymatic metabolism and they compete with endogenous interstitial aden- osine for the transporter, thereby increasing adenosine's extracellular concentration. Because the latter characteristics also apply to the $\mathrm{Al}$ receptor-selective agonist $\mathrm{CHA}$, it should exhibit a greater potency than adenosine if arteriolar Al receptors are involved in the vasodilatative response; however, our observation that $\mathrm{CHA}$ was equipotent with adenosine lends further support to our contention that $\mathrm{A} 2$ receptors are the primary adenosine receptor subtype in the retinal microcirculation. A1 adenosine receptors are virtually absent from cerebral microvessels $(14,17)$, consistent with our findings in the retinal vasculature. Rather, cerebral Al receptors are found heterogeneously distributed on neuronal cell bodies and synapses (13). Similarly, retinal A1 receptors have been localized primarily on cell bodies in the ganglion cell and inner plexiform layers (see [18] for review).

The presence of neuronal and glial adenosine receptors in the retina (18) raises a caveat: Because the retina vasculature, like that of the brain, lies within neural tissue, activation of these nonvascular receptors by exogenously administered adenosine or the other agonists could, indirectly, affect the vascular responses we observed. Because adenosine is likely to induce inhibitory effects on neuronal metabolism (18), a metabolically coupled decrease in RBF might be elicited. This would be particularly applicable to the AI agonists like CHA, because AI receptors, distributed primarily in the inner retina, transduce inhibitory neuromodulatory actions of adenosine; nonvascular actions induced by $\mathrm{A} 2$ agonists may not be as significant, because inner retina A2 receptors are relatively sparse by comparison (18). In any case, it should be cautioned that measurements of changes in RBF or vessel diameter after administration of particular adenosinergic compounds may reflect, in part, these indirect effects and, unfortunately, one cannot identify from these resultant vascular responses how significant this contribution might be. An additional point can be added regarding the distribution of A2 adenosine receptors along the vascular tree. Our observations of arteriolar and venular dilations in response to adenosine agonists do not necessarily indicate the presence of venular A2 receptors; because the retinal vasculature is composed of "end arteries" (6), drug-induced vasodilation in an input arteriole would be expected to cause vasodilation in the output venule by hemodynamic forces alone, independent of receptor-mediated relaxation. Although it is possible that venular adenosine receptors exist, given that the primary site of tissue blood flow control 
is at the arteriolar level, we infer that adenosine increases RBF as a result of its vasodilatative effect on arteriolar A2 receptors.

Having demonstrated vascular reactivity to exogenous adenosine and its agonists, we intended to provide more persuasive evidence of adenosine-based $\mathrm{RBF}$ regulation by documenting vasodilatative responses to increases in endogenous adenosine concentrations. The potent vasodilatative effects of the adenosine kinase inhibitor ITC and the adenosine reuptake blocker NBTI that we observed in the piglet retinal microcirculation indeed support our hypothesis that elevations in endogenous adenosine levels can activate microvascular receptors and thereby elicit an increase in RBF. It was not unexpected that inhibition of adenosine rephosphorylation to AMP would potentiate interstitial adenosine concentrations, because a high-affinity uptake system for adenosine is present in neurons, glia, and endothelial cells of CNS tissue and has been studied in some detail in retina (18). In fact, it has been shown that $85 \%$ of radiolabeled adenosine $(0.6$ $\mathrm{nmol}$ ) is converted via the kinase into adenine nucleotides within $4 \mathrm{~h}$ of its intravitreal administration in rabbits (19); even after $15 \mathrm{~min}$, incorporated label can be identified in ganglion cells (M. Perez, personal communication). In light of these data, it is surprising that vasoactive effects induced by intravitreal administration of adenosine in the rabbit can be of any significant magnitude when measured $6 \mathrm{~h}$ postinjection (3). In any case, although there are no studies of the effects of ITC on adenosine metabolism or compartmentation in the retina, it has been reported that $10 \mu \mathrm{M} \mathrm{ITC}$, the concentration of ITC injected intravitreally in our study, inhibits by $90 \%$ the incorporation of adenosine into cellular nucleotides in both myocardial cells and polymorphonuclear leukocytes (20) and that ITC increases interstitial adenosine concentrations (and cerebral blood flow) in the brain (21). Thus, it is highly likely that the vasodilatative response we noted after ITC administration resulted from an ITC-induced augmentation of interstitial levels of endogenous adenosine. Based on the duration of the ITC-induced vasodilation, which rivaled that seen with the highest doses of adenosine and NECA, a long-lasting inhibition of the kinase was evidenced; a pharmacokinetic explanation may underlie this observation, as the drug functions at an intracellular site of action.

Our documentation of NBTI-induced vasodilation in the piglet retinal microcirculation is consistent with observations in the rabbit retina that retinal vein diameter (3) and RBF [measured by microspheres (4)] increased after an intravitreal injection of dipyridamole, another drug that inhibits adenosine reuptake. We, too, have observed retinal arteriolar and venular dilations after intravitreal microsuffusion of dipyridamole $(0.2 \mathrm{nmol}$; unpublished observations). Our belief that the retinal vasodilation induced by NBTI administration was mediated indirectly through its potentiation of interstitial adenosine concentrations gains support in light of the studies directly demonstrating NBTI inhibition of adenosine uptake in isolated rabbit retinas (9), cultures of chick embryo retinal neurons (10), and cerebral microvessels from the pig (22). More conclusive evidence, however, was provided in the present study: Virtually complete attenuation of NBTI-induced dilation could be achieved with adenosine receptor blockade by the preferential A2 adenosine receptor antagonist DPMX (23). Thus, the dilation we observed had to be mediated by increases in endogenous adenosine concentrations. Adenosine antagonists were also found to attenuate increases in RBF (4) or retinal vein diameter (3) elicited by intravitreal adenosine in rabbits. Moreover, decreases in the velocity of leukocytes in the human macula, as measured by the blue entopic phenomenon, followed the ingestion of caffeine, a well-known adenosine receptor antagonist (24).

Taken together, all these results are consistent with our hypothesis that the metabolite adenosine, whose production is linked to ATP metabolism, serves to increase RBF under various conditions in which an increased substrate supply is required. Indeed, several studies have documented calcium-dependent retinal adenosine release in response to potassium-induced depolar- ization (10, 25), excitatory neurotransmitters (26), and light flashes (25), all conditions associated with increased tissue metabolism. Conversely, retinal adenosine release has been noted to increase in response to hypoxia (27), wherein substrate supply becomes limited. In both cases, the metabolite adenosine is ideally suited to mediate flow-metabolism coupling in the retina. Evidence that, under steady state conditions, adenosine is tonically present in the interstitial fluid comes from studies in which deamination of endogenous adenosine with adenosine deaminase affected neuromodulatory functions (28) or cAMP levels (29) in a manner similar to that caused by adenosine receptor antagonism. Our finding that adenosine receptor blockade with DPMX under baseline conditions did not constrict arterioles and venules does not invalidate the hypothesis for the regulation of RBF by adenosine, because the threshold concentration for vasodilation could be just above normal resting levels of interstitial adenosine. We contend that the concentration of DPMX used should have unmasked any tonic vasodilatative effect of endogenous adenosine, if present, because we could demonstrate a complete reversal of endogenous adenosine-induced vasodilations secondary to NBTI administration with the same dose of DPMX.

Although it might be argued that the loss of endogenous intraocular pressure, as a result of the lensectomy procedure we used to permit high-magnification viewing of the retinal microcirculation, might affect the validity of the present results, we do not feel our methodology reduces the relevance of our findings for the following reasons: We documented that the retinal microcirculation in our preparation dilates in response to hypoxia, hypercapnia, and reductions in retinal perfusion pressure and constricts in response to hyperoxia and hypocapnia, as one would predict based on dozens of previous empirical studies of RBF in several different animal models including the pig $(30,31)$ and piglet (32). This is not to say that the absolute magnitude of the observed vasoactive responses may have been affected slightly by the change in transmural pressure, but the overall validity of our results is difficult to dispute on these grounds.

The recent studies from Campochiaro's laboratory $(3,33)$ that intravitreal injection of either adenosine plus the uptake blocker dipyridamole or the adenosine A2 agonist NECA caused hemorrhages and breakdown of the blood retinal barrier require comment. First of all, we observed no signs of hemorrhage in our preparation after intravitreal administration of up to 200 nmol of adenosine (5 times less than their dose) or $20 \mathrm{pmol}$ of NECA (5000 times less than their dose); nevertheless, these concentrations induced venular dilations in our preparation that were greater than those observed $b_{y} ;$ Campochiaro and Sen (3). We contend that the amount of adenosine injected in their studies $(3,33)$, especially when delivered concomitantly with a high concentration of the uptake blocker dipyridamole (3), resulted in retinal adenosine concentrations significantly greater than those expected during complete tissue ischemia, one of the most potent stimuli known for adenosine production and release. Thus, even if their administered adenosine dose $(1 \mu \mathrm{mol})$ was diluted throughout the vitreous [estimated volume $=1 \mathrm{~mL}(9)$ ], the resultant adenosine concentration would be $1 \mathrm{mM}$, more than an order of magnitude higher than levels measured in other CNS tissue under totally ischemic conditions (34-36). With concomitant adenosine uptake blockade by high concentrations $(0.1 \mathrm{mM})$ of dipyridamole, it is possible that extracellular adenosine levels may have increased even higher still, not to mention the complicating effect of phosphodiesterase inhibition (37) likely to be induced by $0.1 \mathrm{mM}$ dipyridamole. Similarly, although their dose of NECA was 10-fold less than that of adenosine, its effects with respect to adenosine receptor stimulation are questionable, given that the vasodilatative potency of this agonist is 500- to 1000 -fold greater than that of adenosine $(16,38)$; in the present study in the piglet, NECA was 5000 -fold more potent in inducing arteriolar dilation relative to adenosine. Thus, we feel the pathophysiologic relevance of the hemorrhagic effects attributed to 
endogenous adenosine by Campochiaro and Sen should be interpreted with much caution $(3,33)$.

In summary, dose-dependent dilations of retinal arterioles and venules were measured in response to intravitreal microsuffusions of adenosine and its agonists; the resultant potency differences of these agonists indicate that adenosine receptors of the A2 subtype mediate these vasodilatative responses. Pharmacologically induced increases in endogenous interstitial adenosine concentrations by ITC and NBTI also elicited dilations of the retinal microcirculation. Resting levels of interstitial adenosine may not be high enough to affect arteriolar tone in a tonic fashion, however, because adenosine receptor blockade with DPMX failed to constrict these vessels. Our findings are consistent with the hypothesis that adenosine is a metabolic regulator of $\mathrm{RBF}$ in the eye of the newborn. Experiments undertaken in our laboratory to test this hypothesis directly, wherein the effects of specific adenosine antagonists on RBF are assessed under conditions of increased RBF induced by physiologically relevant stimuli, have recently been published (39).

Acknowledgments. The authors are thankful for the invaluable assistance of our technical staff: Tina Lanius, Aarti Shah, and Ernesto Gonzales.

\section{REFERENCES}

1. Bill A, Sperber GO 1990 Control of retinal and choroidal blood flow. Eye $4: 319-325$

2. Phillis JW 1989 Adenosine in the control of the cerebral circulation. Cerebrovasc Brain Metab Rev 1:26-54

3. Campochiaro PA. Sen HA 1989 Adenosine and its agonists cause retinal vasodilation and hemorrhages. Implications for ischemic retinopathies. Arch Ophthalmol 107:412-416

4. Braunagel SC. Xiao JG, Chiou GCY 1988 The potential role of adenosine in regulating blood flow in the eye. $\mathrm{J}$ Ocular Pharmacol 4:61-73

5. Flower RW, Patz A 1971 Oxygen studies in retrolental fibroplasia. IX. The effects of elevated arterial oxygen tension on retinal vascular dynamics in the kitten. Arch Ophthalmol 85:197-203

6. Rootman J 1971 Vascular system of the optic nerve head and retina of the pig. Br J Ophthalmol 55:808-819

7. Winn HR. Park TS. Curnish RR, Rubio R. Berne RM 1980 Incorporation of adenosine and its metabolites into brain nucleotides. Am J Physiol 239:H212-H219

8. Wu PH, Phillis JW 1982 Uptake of adenosine by isolated rat brain capillaries J Neurochem 38:687-690

9. Blazynski $\mathrm{C} 1991$ The accumulation of $\left[{ }^{3} \mathrm{H}\right]$ phenylisopropyl adenosine $\left(\left[{ }^{3} \mathrm{H}\right]\right.$ $\mathrm{PIA})$ and $\left[{ }^{3} \mathrm{H}\right] \mathrm{adenosine}$ into rabbit retinal neurons is inhibited by nitrobenzylthioinosine (NBI). Neurosci Lett 121:1-4

10. Paes de Carvalho R. Braas KM. Snyder SH, Adler R 1990 Analysis of adenosine immunoreactivity. uptake, and release in purified cultures of developing chick embryo retinal neurons and photoreceptors. J Neurochem 55:16031611

11. Ehinger B. Perez MTR 1984 Autoradiography of nucleoside uptake into the retina. Neurochem Int 6:369-381

12. Senba E. Daddona PE. Nagy JI 1986 Immunohistochemical localization of adenosine deaminase in the retina of the rat. Brain Res Bull 17:209-217

13. Reddington M, Lee KS 1991 Adenosine receptor subtypes: classification and distribution. In: Stone TW (ed) Adenosine in the Nervous System. Academic Press, New York, pp 77-118

14. Kalaria RN. Harik SI 1986 Adenosine receptors of cerebral microvessels and choroid plexus. J Cereb Blood Flow Metab 6:463-470
15. Laudignon N. Aranda JV, Varma DR 1990 Effect of adenosine and its analogues on isolated internal carotid arteries from newborn and adult pigs. Biol Neonate 58:91-97

16. Ibayashi S, Ngai AC. Meno JR, Winn HR 1990 Effects of topical adenosine analogs and forskolin on rat pial arterioles in vivo. J Cereb Blood Flow Metab 11:72-76

17. Li YO, Fredholm BB 1985 Adenosine analogues stimulate cyclic AMP formation in rabbit cerebral microvessels via adenosine A2-receptors. Acta Physiol Scand 124:253-259

18. Blazynski C. Perez M-TR 1991 Adenosine in vertebrate retina: localization, receptor characterization, and function. Cell Mol Neurobiol 11:463-484

19. Perez MTR, Ehinger B, Lindström K. Fredholm BB 1986 Release of endogenous and radioactive purines from the rabbit retina. Brain Res 398:106-112

20. Newby AC, Holmquist CA, Illingworth JA, Pearson JD 1983 The control of adenosine concentration in polymorphonuclear leukocytes, cultured heart cells, and isolated perfused heart from the rat. Biochem J 214:317-323

21. Sciotti VM, Van Wylen DGL 1993 Increases in interstitial adenosine and cerebral blood flow with inhibition of adenosine kinase and adenosine deaminase. J Cereb Blood Flow Metab 13:201-207

22. Kalaria RN. Harik SI 1986 Nucleoside transporter of cerebral microvesseis and choroid plexus. J Neurochem 47:1849-1856

23. Ukena D, Shamin MT, Padgett W, Daly JW 1986 Analogs of caffeine: antagonists with selectivity for A2 adenosine receptors. Life Sci 39:743-750

24. Lotfi K, Grunwald JE 1991 The effect of caffeine on the human macular circulation. Invest Ophthalmol Vis Sci 32:3028-3032

25. Perez MTR, Arner K. Ehinger B 1988 Stimulation-evoked release of purines from the rabbit retina. Neurochem Int 13:307-318

26. Perez MTR. Ehinger B 1989 Multiple neurotransmitter systems influence the release of adenosine derivatives from the rabbit retina. Neurochem Int $15: 411-420$

27. DeBenedetto R, Crosson CE 1992 The relatively selective Al adenosine agonist cyclopentyladenosine suppresses dopamine release in bovine retina. Invest Ophthalmol Vis Sci 33:1405(abstr)

28. Blazynski C. Woods C, Mathews GC 1992 Evidence for the action of endogenous adenosine in the rabbit retina: modulation of the light-evoked release of acetylcholine. J Neurochem 58:761-767

29. Paes de Carvalho R, de Mello FG 1982 Adenosine-elicited accumulation of adenosine $3^{\prime}, 5^{\prime}$-cyclic monophosphate in the chick embryo retina. $\mathrm{J}$ Neurochem 38:493-500

30. Riva CE, Pournaras CJ, Tsacopoulos M 1986 Regulation of local oxygen tension and blood flow in the inner retina during hyperoxia. $J$ Appl Physiol 1986:61:592-598

31. Ffytche TJ, Bulpitt CJ, Kohner EM, Archer D, Dollery CT 1974 Effect of changes in intraocular pressure on the retinal microcirculation. $\mathrm{Br} \mathrm{J}$ Ophthalmol 58:514-522

32. Stiris T, Odden JP, Hansen TW, Hall C, Bratlid D 1989 The effect of arterial $\mathrm{PCO}_{2}$-variations on ocular and cerebral blood flow in the newborn piglet. Pediatr Res 25:205-208

33. Sen HA, Campochiaro PA 1989 Intravitreous injection of adenosine or its agonists causes breakdown of the blood-retinal barrier. Arch Ophthalmol 107:1364-1367

34. Park TS. Gidday JM 1990 Effect of dipyridamole on cerebral extracellular adenosine level in vivo. J Cereb Blood Flow Metab 10:424-427

35. Van Wylen DGL, Park TS, Rubio R, Berne RM 1986 Increases in cerebral interstitial fluid adenosine concentration during hypoxia, local potassium infusion, and ischemia. J Cereb Blood Flow Metab 6:522-528

36. Hagberg H, Andersson P. Lacarewicz J, Jacobson I, Butcher S, Sandberg M 1987 Extracellular adenosine, inosine, hypoxanthine, and xanthine in relation to tissue nucleotides and purines in rat striatum during transient ischemia. J Neurochem 49:227-231

37. FitzGerald GA 1987 Dipyridamole. N Engl J Med 316:1247-1255

38. Van Wylen DGL, Park TS, Rubio R, Berne RM 1989 The effect of local infusion of adenosine and adenosine analogues on local cerebral blood flow. $\mathrm{J}$ Cereb Blood Flow Metab 9:556-562

39. Gidday JM, Park TS 1993 Adenosine-mediated autoregulation of retinal arteriolar tone in the piglet. Invest Ophthalmol Vis Sci 34 (in press) 